\title{
Primary resonance of traveling viscoelastic beam under internal resonance*
}

\author{
Hu DING ${ }^{1, \dagger}$, Linglu HUANG ${ }^{1}$, Xiaoye MAO ${ }^{1}$, Liqun CHEN ${ }^{1,2}$ \\ 1. Shanghai Institute of Applied Mathematics and Mechanics, Shanghai Key Laboratory of \\ Mechanics in Energy Engineering, Shanghai University, Shanghai 200072, China; \\ 2. Department of Mechanics, College of Science, Shanghai University, Shanghai 200444, China
}

\begin{abstract}
Under the 3:1 internal resonance condition, the steady-state periodic response of the forced vibration of a traveling viscoelastic beam is studied. The viscoelastic behaviors of the traveling beam are described by the standard linear solid model, and the material time derivative is adopted in the viscoelastic constitutive relation. The direct multi-scale method is used to derive the relationships between the excitation frequency and the response amplitudes. For the first time, the real modal functions are employed to analytically investigate the periodic response of the axially traveling beam. The undetermined coefficient method is used to approximately establish the real modal functions. The approximate analytical results are confirmed by the Galerkin truncation. Numerical examples are presented to highlight the effects of the viscoelastic behaviors on the steady-state periodic responses. To illustrate the effect of the internal resonance, the energy transfer between the internal resonance modes and the saturation-like phenomena in the steady-state responses is presented.
\end{abstract}

Key words traveling beam, nonlinear vibration, viscoelasticity, primary resonance, internal resonance

Chinese Library Classification O242

2010 Mathematics Subject Classification 74S05

\section{Introduction}

This paper presents a profound study for the nonlinear forced vibration of an axially traveling beam with 3:1 internal resonance. The main target of the present paper is to investigate the effects of the viscoelastic properties and the internal resonance on the steady-state response of the traveling beam. Axially traveling beams play an important role in the design of modern machinery. Therefore, the linear and nonlinear dynamics of the axially traveling beams have drawn many researchers' attention ${ }^{[1-3]}$. Marynowski and Kapitaniak ${ }^{[4]}$ have reviewed the research progress of the dynamics of the axially traveling continua in the past sixty years.

Traditionally, the investigations were focused on the dynamics of the traveling elastic beams. Very recently, researchers have paid their attention on the viscoelastic behaviors of the traveling beam. Yao and Zhang ${ }^{[5]}$ established the equations of motion for the viscoelastic moving belt

* Received May 20, 2016 / Revised May 30, 2016

Project supported by the State Key Program of the National Natural Science Foundation of China

(No. 11232009) and the National Natural Science Foundation of China (Nos. 11372171 and 11422214)

$\dagger$ Corresponding author, E-mail: dinghu3@shu.edu.cn 
by use of the Kelvin-type viscoelastic constitutive law. Ozhan and Pakdemirli ${ }^{[6-7]}$ and Yang and Zhang ${ }^{[8]}$ adopted the Kelvin model containing the partial time derivative for describing the viscoelastic behavior of beam materials. To study the nonlinear vibration of the traveling viscoelastic beams, Ding and $\mathrm{Zu}^{[9]}$, Yan et al. ${ }^{[10]}$, and Tang et al. ${ }^{[11]}$ proved that the material time derivative should be contained in the Kelvin model. The standard linear solid model has been employed in modelling axially traveling viscoelastic beams. Wang et al. ${ }^{[12]}$ investigated the effect of an arbitrary varying length on the nonlinear free vibration of an axially translating viscoelastic beam. Chen and his co-workers worked on the steady-state responses of the axially traveling strings ${ }^{[13]}$ and beams ${ }^{[14]}$ with the standard linear solid model. Saksa and Jeronen ${ }^{[15]}$ studied the characteristic behaviors of an axially traveling viscoelastic beam by modelling the viscoelasticity with the Poynting Thomson version of the standard linear solid. In the present paper, the effect of the standard linear solid model on the nonlinear dynamics of the traveling beams with internal resonance is firstly investigated.

Generally, internal resonance can complicate the dynamics of the nonlinear system. Therefore, the nonlinear vibration of the traveling beam under internal resonance has been widely concerned. With the Galerkin method, Ghayesh and Amabili ${ }^{[16]}$ tuned the first two transverse modes to a 3:1 internal resonance, and studied the coupled longitudinal and transverse vibration of an axially traveling beam. By combining the incremental harmonic balance method with the Galerkin method, Chen and his co-workers discovered the planar motion ${ }^{[17]}$ and the nonlinear vibration to periodic lateral force excitations ${ }^{[18]}$ for an axially traveling beam. Wang et al. ${ }^{[19]}$ considered the multimode dynamics of the inextensional beams on the elastic foundation with 2:1 internal resonance based on a similar process. Since the governing equations are discretized into ordinary differential equations, the research techniques in the above-mentioned literatures are called as the indirect perturbation method. The nonlinear dynamics of the traveling beams have also been studied by the direct perturbation method. By use of the direct multi-scale method, Sahoo et al. ${ }^{[20]}$ focused on the nonlinear transverse vibration of an axially moving beam subjected to two frequency excitations in the presence of internal resonance. By use of the direct multi-scale method to establish the solvability conditions, Tang et al..11] investigated the parametric and 3:1 internal resonance of the axially moving viscoelastic beams on the elastic foundation. Without internal resonance, the multi-scale method has been directly used to solve the nonlinear dynamics of the traveling beams. Forced vibrations are studied for traveling beams via the direct multi-scale analysis ${ }^{[21]}$. Liu et al. ${ }^{[22]}$ derived the analytical expression of the first-order uniform expansion of the solution by directly using the multi-scale method. One thing should be mentioned, by use of the direct multi-scale method to study the nonlinear dynamics of traveling beams, all the above-mentioned studies establish the solvability conditions with complex modal functions. The reason is that it is difficult to obtain the real modal functions for the derived equation of the nonlinear continuous Gyro system.

In the present work, the forced periodic response of an axially traveling viscoelastic beam is investigated in the presence of 3:1 internal resonance. The standard linear solid model and the material time derivative are used to model the transverse vibration of the axially traveling beam. The effects of the viscoelastic property on the periodic response are determined. The approximate real modal functions are established based on the corresponding linear derived equation. Therefore, the direct multi-scale method is firstly used to establish the solvable conditions with the real modal functions. The approximate analytical stable steady-state response is confirmed by the Galerkin method.

\section{Mathematical model}

Consider a slender axially traveling beam with simply supported boundary conditions (see Fig. 1). The traveling beam is modelled as an Euler-Bernoulli viscoelastic beam. The transverse vibration of the traveling beam with the external harmonic excitation is governed 


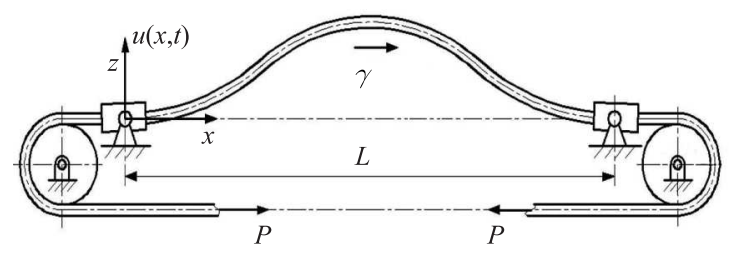

Fig. 1 Traveling beam with simply supported ends

by ${ }^{[23]}$

$$
\rho A\left(u, t t+\dot{\gamma} u,_{x}+2 \gamma u,_{x t}+\gamma^{2} u,_{x x}\right)+M,_{x x}=\left((P+A \sigma) u,_{x}\right),{ }_{x}+F,
$$

where $t$ is the time, and $x$ is the neutral axis coordinate along the traveling beam. A comma preceding $t$ or $x$ denotes partial derivatives. $u(x, t)$ presents the transverse displacement. Moreover, $L$ and $A$ are, respectively, the length and the cross-section area of the beam. $F=b \sin (\omega t)$ denotes the transverse load, where $b$ and $\omega$ are the excitation amplitude and the frequency, respectively. $P$ is the initial axial load. $\sigma(x, t)$ presents the disturbed axial stress. For a slender traveling beam, the bending moment $M(x, t)$ is expressed as follows:

$$
M(x, t)=-\int_{\mathrm{A}} z \sigma(x, t) \mathrm{d} A .
$$

The standard linear solid model with the material time derivative is used to characterize the viscoelastic property of the traveling beam. Therefore, the stress-strain relation is expressed in a differential form as follows:

$$
\left(E_{1}+E_{2}\right) \sigma+\alpha \frac{\mathrm{d} \sigma}{\mathrm{d} t}=E_{1} E_{2} \varepsilon_{\mathrm{L}}+E_{1} \alpha \frac{\mathrm{d} \varepsilon_{\mathrm{L}}}{\mathrm{d} t},
$$

where $E_{1}$ and $E_{2}$ are the stiffness constants, $\sigma$ denotes the normal stress due to that bending $\alpha$ represents the dynamic viscosity, and $\varepsilon_{\mathrm{L}}(x, t)$ is the Lagrangian axial bending strain expressed by

$$
\varepsilon_{\mathrm{L}}(x, t)=\frac{1}{2} u^{2},{ }_{x} .
$$

The total time derivative is given by

$$
\frac{\mathrm{d}}{\mathrm{d} t}=\frac{\partial}{\partial t}+\gamma \frac{\partial}{\partial x}
$$

Therefore, substituting Eqs. (4) and (5) into Eq. (3) yields

$$
\left(E_{1}+E_{2}\right) \sigma+\alpha \sigma,_{t}+\alpha \gamma \sigma,_{x}=\frac{1}{2}\left(E_{1} E_{2}\left(u^{2},{ }_{x}\right)+E_{1} \alpha\left(u^{2},_{x}\right){ }_{,}+E_{1} \alpha \gamma\left(u^{2},{ }_{x}\right){ }_{x}\right) .
$$

In this paper, only small deflections are considered. Therefore, the displacement-strain relationship can be written as follows:

$$
\varepsilon_{\mathrm{S}}(x, z, t)=-z u,_{x x}
$$

Therefore, for the standard linear solid, the viscoelastic material of the beam has the following constitution relationship:

$$
\left(E_{1}+E_{2}\right) M+\alpha M,_{t}+\alpha \gamma M,_{x}=\left(E_{1} E_{2} u,_{x x}+E_{1} \alpha u,,_{x x t}+E_{1} \alpha \gamma u, x x x\right) \int_{A} z^{2} \mathrm{~d} A
$$


Due to bending and the bending moment, the normal stresses are, respectively, derived from Eqs. (6) and (8). In the following investigation, the simply supported boundary conditions of the traveling beam are considered as follows:

$$
u(0, t)=u(L, t)=0, \quad u,_{x x}(0, t)=u,_{x x}(L, t)=0 .
$$

Incorporate the following dimensionless variables and parameters:

$$
\left\{\begin{array}{l}
u \leftrightarrow \frac{u}{L}, \quad x \leftrightarrow \frac{x}{L}, \quad t \leftrightarrow t \sqrt{\frac{P}{\rho A L^{2}}} \\
\gamma \leftrightarrow \gamma \sqrt{\frac{\rho A}{P}}, \quad \alpha \leftrightarrow \frac{\alpha}{E_{1}+E_{2}} \sqrt{\frac{P}{\rho A L^{2}}}, \quad \omega \leftrightarrow \omega \sqrt{\frac{\rho A L^{2}}{P}} \\
b \leftrightarrow \frac{b L}{P}, \quad I=\int_{A} z^{2} \mathrm{~d} A, \quad E_{a}=\frac{I E_{1} E_{2}}{P L^{2}\left(E_{1}+E_{2}\right)} \\
E_{b}=\frac{I E_{1}}{P L^{2}}, \quad E_{c}=\frac{E_{1} E_{2} A}{P\left(E_{1}+E_{2}\right)}, \quad E_{d}=\frac{E_{1} A}{P}
\end{array}\right.
$$

where $I$ denotes the area moment of inertial, $\alpha$ denotes the dimensionless viscous coefficient, $E_{a}$ and $E_{b}$ are, respectively, represent the effects of the flexural stiffness of the traveling beam, and $E_{c}$ and $E_{d}$ are the nonlinear coefficients. The governing equation of the transverse vibration of the traveling beam and the boundary conditions are nondimensionalized as follows:

$$
\begin{aligned}
& u,_{t t}+2 \gamma u,_{x t}+\left(\gamma^{2}-1\right) u,_{x x}+E_{a} u,_{x x x x}+\alpha\left(E_{b}-E_{a}\right)\left(u,_{x x x x t}+\gamma u,_{x x x x x}\right) \\
= & \frac{3}{2} E_{c} u,_{x}^{2} u,_{x x}-\alpha u,_{x}\left(E_{c}-E_{d}\right)\left(2 u,_{x x}\left(u,_{x t}+\gamma u,_{x x}\right)\right. \\
& \left.+u,_{x}\left(u,_{x x t}+\gamma u,_{x x x}\right)\right)+b \sin (\omega t), \\
& u(0, t)=u(1, t)=0, \quad u,_{x x}(0, t)=u,_{x x}(1, t)=0 .
\end{aligned}
$$

\section{Natural frequencies and internal resonance condition}

To construct the condition of the internal resonance of the traveling beam, the corresponding linear equation can be adopted to abstract the natural frequencies of the traveling beam. Omitting the nonlinear terms and viscosity terms of Eq. (11) yields

$$
u, t t+2 \gamma u,_{x t}+\left(\gamma^{2}-1\right) u,_{x x}+E_{a} u,_{x x x x}=0 .
$$

The solution to the linear derived equation (13) can be expressed as follows:

$$
u(x, t)=\sum_{n=1}^{+\infty} \Phi_{n}(x) \mathrm{e}^{\mathrm{i} \omega_{n} t}+\text { c.c. }, \quad n=1,2, \cdots
$$

where c.c. represents the complex conjugate of all preceding terms on the right-hand side of the equation, and the modal function is assumed as follows:

$$
\Phi_{n}(x)=\sum_{m=1}^{+\infty} c_{n, m} \sin (m \pi x), \quad \overline{\Phi_{n}}(x)=\sum_{m=1}^{+\infty} \overline{c_{n, m}} \sin (m \pi x) .
$$


Substitute Eqs. (14) and (15) into Eq. (13). Then, multiplying both sides of the obtained equation by $\sin (m \pi x)(m=1,2, \cdots)$ and integrating it with respect to $x$ from 0 to 1 yield a set of second-order ordinary differential equations. The non-triviality of the solutions to the set of the ordinary differential equations requires its determinant of coefficients to be zero. Therefore, the following equation is obtained:

$$
\left|-\omega_{n}^{2} \boldsymbol{M}+\mathrm{i} \omega_{n} \boldsymbol{Z}+\boldsymbol{K}\right|=0,
$$

where $\boldsymbol{M}$ represents the identity matrix, and $\boldsymbol{Z}$ and $\boldsymbol{K}$ are, respectively, the Coriolis acceleration matrix and the stiffness matrix defined by

$$
\begin{aligned}
& Z_{i j}=4 \gamma\left\{\begin{array}{l}
\frac{i j\left(1-(-1)^{i+j}\right)}{i^{2}-j^{2}}, \quad i \neq j, \\
0, \quad i=j,
\end{array}\right. \\
& K_{i j}= \begin{cases}i^{4} \pi^{4} E_{a}+i^{2} \pi^{2}\left(1-\gamma^{2}\right), \quad i=j, \\
0, \quad i \neq j .\end{cases}
\end{aligned}
$$

The natural frequencies $\omega_{n}$ can be obtained from Eq. (16).

In the following study, a V-belt is adopted as the prototype of the traveling beam. Figure 2 shows the shape of the cross-sectional area of the V-belt. Table 1 presents the physical parameters of the V-belt.

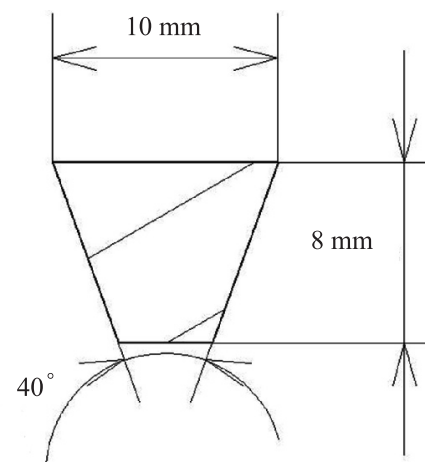

Fig. 2 Shape of cross-sectional area of V-belt

Table 1 Physical parameters of V-belt transmission

\begin{tabular}{ccc}
\hline Item & Notation & Value \\
\hline Cross-section area & $A$ & $5.671 \times 10^{-5} \mathrm{~m}^{2}$ \\
Area moment of inertial & $I$ & $2.775 \times 10^{-9} \mathrm{~m}^{4}$ \\
Initial axial tension & $P$ & $20 \mathrm{~N}$ \\
Length of belt & $L$ & $0.35 \mathrm{~m}$ \\
Modulus of elasticity & $E_{1}$ & $200 \mathrm{MPa}$ \\
Creep modulus of elasticity & $E_{2}$ & $200 \mathrm{MPa}$ \\
\hline
\end{tabular}

Based on Table 1, the dimensionless parameters can be determined based on Eq. (10). Therefore,

$$
E_{b}=2 E_{a}=0.058, \quad E_{d}=2 E_{c}=567.2, \quad b=0.003, \quad \alpha=0.005 .
$$

If there is no special designation, the traveling beam can be considered with these parameter values in all the following examples. The first four natural frequencies are calculated and shown 
in Table 2 with the dimensionless axial speed $\gamma=0.598$ 29. As shown in Table 2 , the internal resonance condition $\omega_{2}: \omega_{1}=3$ is satisfied.

Table 2 First four natural frequencies of derived linear equation

\begin{tabular}{ccc}
\hline Item & Notation & Value \\
\hline First natural frequency & $\omega_{1}$ & 2.79792 \\
Second natural frequency & $\omega_{2}$ & 8.39377 \\
Third natural frequency & $\omega_{3}$ & 17.12350 \\
Fourth natural frequency & $\omega_{4}$ & 30.38160 \\
\hline
\end{tabular}

\section{Scheme of approximate analytical solution}

In this section, the multi-scale method is used to solve the steady-state periodic responses of the axially traveling beam. In this work, only weak external excitation is considered. A non-dimensional bookkeeping parameter is introduced to distinguish the different orders of magnitude. Therefore, the following dimensionless variable and parameters are scaled:

$$
b \leftrightarrow \varepsilon^{3} b, \quad \alpha \leftrightarrow \varepsilon^{2} \alpha, \quad u(x, t) \leftrightarrow \varepsilon u(x, t) .
$$

Then, the perturbation solution can be written as follows:

$$
u\left(x, T_{0}, T_{2}\right)=u_{0}\left(x, T_{0}, T_{2}\right)+\varepsilon^{2} u_{2}\left(x, T_{0}, T_{2}\right),
$$

where $T_{0}=t, T_{2}=\varepsilon^{2} t$, and the following relationships are satisfied:

$$
\frac{\partial}{\partial t}=\frac{\partial}{\partial T_{0}}+\varepsilon^{2} \frac{\partial}{\partial T_{2}}, \quad \frac{\partial^{2}}{\partial t^{2}}=\frac{\partial^{2}}{\partial T_{0}^{2}}+2 \varepsilon^{2} \frac{\partial^{2}}{\partial T_{0} \partial T_{2}}+\varepsilon^{4} \frac{\partial^{2}}{\partial T_{2}^{2}} .
$$

Substituting Eqs. (19), (20), and (21) into Eq. (11) and equalizing the coefficients of $\varepsilon^{0}$ and $\varepsilon^{2}$ in the obtained equations yield

$$
\begin{aligned}
& u_{0, T_{0} T_{0}}+E_{a} u_{0, x x x x}+2 \gamma u_{0, x T_{0}}+u_{0, x x} \gamma^{2}-u_{0, x x}=0, \\
& 2 u_{0, T_{0} T_{2}}+u_{2, T_{0} T_{0}}+2 \gamma u_{2, x T_{0}}+2 \gamma u_{0, x T_{2}}+\gamma^{2} u_{2, x x}+\alpha \gamma\left(E_{b}-E_{a}\right) u_{0, x x x x x} \\
& -3 E_{c} u_{0, x}^{2} u_{0, x x} / 2+\alpha\left(E_{b}-E_{a}\right) u_{0, x x x x T_{0}}+E_{a} u_{2, x x x x}-u_{2, x x}+b \sin (\omega t)=0 .
\end{aligned}
$$

The solution of Eq. (22) can be written as follows:

$$
u_{0}\left(x, T_{0}, T_{2}\right)=A_{1}\left(T_{2}\right) \Theta_{1}(x) \mathrm{e}^{\mathrm{i} \omega_{1} T_{0}}+A_{2}\left(T_{2}\right) \Theta_{2}(x) \mathrm{e}^{\mathrm{i} \omega_{2} T_{0}}+\text { c.c. },
$$

where $A_{1}\left(T_{2}\right)$ and $A_{2}\left(T_{2}\right)$ are undetermined functions, c.c. represents the complex conjugate of the two preceding terms on the right hand of the equation, and

$$
\left\{\begin{array}{l}
\Theta_{k}(x)=p_{k, 1} \sin (\pi x)+p_{k, 2} \sin (2 \pi x)+p_{k, 3} \sin (3 \pi x)+p_{k, 4} \sin (4 \pi x), \\
\overline{\Theta_{k}}(x)=\overline{p_{k, 1}} \sin (\pi x)+\overline{p_{k, 2}} \sin (2 \pi x)+\overline{p_{k, 3}} \sin (3 \pi x)+\overline{p_{k, 4}} \sin (4 \pi x),
\end{array}\right.
$$

where $k=1,2$. Substitute Eqs. (25) and (24) into Eq. (22). Then, $p_{k, r}$ and $\overline{p_{k, r}}(k=1,2$ and $r=1,2,3,4)$ can be solved by the undetermined coefficient method, and the solution of Eq. (23) can be written as follows:

$$
u_{2}\left(x, T_{0}, T_{2}\right)=Q_{1}\left(x, T_{2}\right) \mathrm{e}^{\mathrm{i} \omega_{1} T_{0}}+Q_{2}\left(x, T_{2}\right) \mathrm{e}^{\mathrm{i} \omega_{2} T_{0}},
$$


where

$$
Q_{k}\left(x, T_{2}\right)=q_{k, 1}\left(T_{2}\right) \sin (\pi x)+q_{k, 2}\left(T_{2}\right) \sin (2 \pi x)+q_{k, 3}\left(T_{2}\right) \sin (3 \pi x)+q_{k, 4}\left(T_{2}\right) \sin (4 \pi x) .
$$

Introduce the detuning parameters $\sigma_{1}$ and $\sigma_{2}$. Then, the nearnesses of $\omega$ to $\omega_{1}$ and $\omega_{2}$ to $3 \omega_{1}$ can be, respectively, represented by

$$
\omega_{2}=3 \omega_{1}+\varepsilon^{2} \sigma_{1}, \quad \omega=\omega_{1}+\varepsilon^{2} \sigma_{2} .
$$

Substituting Eqs. (26) and (28) into Eq. (23) yields

$$
\begin{aligned}
& 2\left(\mathrm{i} \omega_{1} A_{1}, T_{2} \Theta_{1} \mathrm{e}^{\mathrm{i} \omega_{1} T_{0}}+\mathrm{i} \omega_{2} A_{2}, T_{2} \Theta_{2} \mathrm{e}^{\mathrm{i} \omega_{2} T_{0}}+\text { c.c. }\right)+2 \gamma\left(A_{1, T_{2}} \Theta_{1, x} \mathrm{e}^{\mathrm{i} \omega_{1} T_{0}}\right. \\
& \left.+A_{2}, T_{2} \Theta_{2, x} \mathrm{e}^{\mathrm{i} \omega_{2} T_{0}}+\text { c.c. }\right)-3 E_{c}\left(A_{1} \Theta_{1, x} \mathrm{e}^{\mathrm{i} \omega_{1} T_{0}}+A_{2} \Theta_{2},{ }_{x} \mathrm{e}^{\mathrm{i} \omega_{2} T_{0}}+\text { c.c. }\right)^{2} \\
& \cdot\left(A_{1} \Theta_{1, x x} \mathrm{e}^{\mathrm{i} \omega_{1} T_{0}}+A_{2} \Theta_{2}, x_{x} \mathrm{e}^{\mathrm{i} \omega_{2} T_{0}}+\text { c.c. }\right) / 2 \\
& -\omega_{1}^{2} Q_{1} \mathrm{e}^{\mathrm{i} \omega_{1} T_{0}}+\alpha\left(E_{b}-E_{a}\right)\left(\mathrm{i} \omega_{1} A_{1} \Theta_{1}, x x x x \mathrm{e}^{\mathrm{i} \omega_{1} T_{0}}+\mathrm{i} \omega_{2} A_{2} \Theta_{2}, x x x x \mathrm{e}^{\mathrm{i} \omega_{2} T_{0}}+\text { c.c. }\right) \\
& +\mathrm{i} b\left(\mathrm{e}^{\mathrm{i} \omega T_{0}}-\mathrm{e}^{-\mathrm{i} \omega T_{0}}\right) / 2-\omega_{2}^{2} Q_{2} \mathrm{e}^{\mathrm{i} \omega_{2} T_{0}}+\alpha\left(E_{b}-E_{a}\right) \gamma\left(A_{1} \Theta_{1, x x x x x} \mathrm{e}^{\mathrm{i} \omega_{1} T_{0}}\right. \\
& \left.+A_{2} \Theta_{2},{ }_{x x x x x} \mathrm{e}^{\mathrm{i} \omega_{2} T_{0}}+\text { c.c. }\right)+\gamma^{2}\left(Q_{1}, x x \mathrm{e}^{\mathrm{i} \omega_{1} T_{0}}+Q_{2},{ }_{x x} \mathrm{e}^{\mathrm{i} \omega_{2} T_{0}}\right)
\end{aligned}
$$

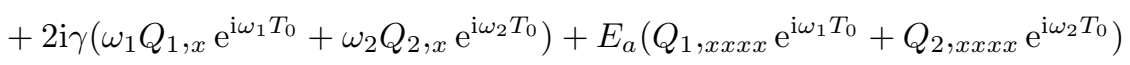

$$
\begin{aligned}
& -\left(Q_{1, x x} \mathrm{e}^{\mathrm{i} \omega_{1} T_{0}}+Q_{2, x x} \mathrm{e}^{\mathrm{i} \omega_{2} T_{0}}\right)=0 .
\end{aligned}
$$

Substituting Eqs.(24), (25), and (27) into Eq. (29), multiplying both sides of the obtained equation by $\sin (m \pi x)(m=1,2,3,4)$, integrating with respect to $x$ from 0 to 1 , and then abstracting the coefficients of $\exp \left(\mathrm{i} \omega_{1} T_{0}\right)$ and $\exp \left(\mathrm{i} \omega_{2} T_{0}\right)$ yield the following equation:

$$
\left(\begin{array}{cccc}
K_{11}-\omega_{k}^{2} & -16 \mathrm{i} \gamma \omega_{k} / 3 & 0 & -32 \mathrm{i} \gamma \omega_{k} / 15 \\
16 \mathrm{i} \gamma \omega_{k} / 3 & K_{22}-\omega_{k}^{2} & -48 \mathrm{i} \gamma \omega_{1} / 5 & 0 \\
0 & 48 \mathrm{i} \gamma \omega_{k} / 5 & K_{33}-\omega_{k}^{2} & -96 \mathrm{i} \gamma \omega_{k} / 7 \\
32 \mathrm{i} \gamma \omega_{k} / 15 & 0 & 96 \mathrm{i} \gamma \omega_{k} / 7 & K_{44}-\omega_{k}^{2}
\end{array}\right)\left(\begin{array}{c}
q_{k, 1} \\
q_{k, 2} \\
q_{k, 3} \\
q_{k, 4}
\end{array}\right)=\left(\begin{array}{c}
H_{k, 1} \\
H_{k, 2} \\
H_{k, 3} \\
H_{k, 4}
\end{array}\right)
$$

where $k=1,2 . K_{11}, K_{22}, K_{33}$, and $K_{44}$ are defined in Eq. (18). $H_{k, 1}, H_{k, 2}, H_{k, 3}$, and $H_{k, 4}$ are defined by

$$
\left\{\begin{aligned}
H_{k, 1}= & \left(B_{1}+8 p_{k, 2} \gamma C_{1} / 3+16 p_{k, 4} \gamma C_{2} / 15+\text { c.c. }\right)+D_{1}\left(A_{k}\left(4 p_{k, 2} / 15\right)+\text { c.c. }\right)^{2}+E, \\
H_{k, 2}= & \left(B_{2}+8 p_{k, 1} \gamma C_{5} / 3-24 p_{k, 3} \gamma C_{6} / 5+\text { c.c. }\right) \\
& +D_{2}\left(A_{1}\left(4 p_{k, 1} / 3+12 p_{k, 3} / 5\right)+\text { c.c. }\right)^{2}+E, \\
H_{k, 3}= & \left(B_{3}+24 p_{k, 2} \gamma C_{3} / 5-48 p_{k, 4} \gamma C_{4} / 7+\text { c.c. }\right) \\
& +D_{3}\left(A_{k}\left(12 p_{k, 2} / 5-24 p_{k, 4} / 7\right)+\text { c.c. }\right)^{2}+E, \\
H_{k, 4}= & \left(B_{4}+16 p_{k, 1} \gamma C_{5} / 15+48 p_{k, 3} \gamma C_{6} / 7+\text { c.c. }\right) \\
& +D_{4}\left(A_{1}\left(8 p_{k, 2} / 15+24 p_{k, 3} / 7\right)+\text { c.c. }\right)^{2}+E,
\end{aligned}\right.
$$


where

$$
\left\{\begin{array}{l}
B_{i}=\mathrm{i} \omega_{1} p_{k, i}\left(A_{1}, T_{2}+(\mathrm{i} \pi)^{4} \alpha\left(E_{b}-E_{a}\right) A_{1}\right), \quad i=1,2,3,4, \quad k=1,2, \\
C_{1}=A_{k}, T_{2}-8 \alpha\left(E_{b}-E_{a}\right) \pi^{4} A_{k}, \quad C_{2}=A_{k}, T_{2}-128 \alpha\left(E_{b}-E_{a}\right) \pi^{4} A_{k}, \\
C_{3}=A_{k}, T_{2}+8 \alpha\left(E_{b}-E_{a}\right) \pi^{4} A_{k}, \quad C_{4}=A_{k}, T_{2}+128 \alpha\left(E_{b}-E_{a}\right) \pi^{4} A_{k}, \\
C_{5}=A_{k}, T_{2}+\alpha\left(E_{b}-E_{a}\right) \pi^{4} A_{k} / 2, \quad C_{6}=A_{k}, T_{2}+81 \alpha\left(E_{b}-E_{a}\right) \pi^{4} A_{k} / 2, \\
E=\mathrm{i} b\left(\mathrm{e}^{\mathrm{i} \omega T_{0}}-\mathrm{e}^{-\mathrm{i} \omega T_{0}}\right) / 2, \quad D_{i}=3 E_{c}\left(i^{2} \pi^{2} A_{k} p_{k, i}+\text { c.c. }\right) / 4 .
\end{array}\right.
$$

Therefore, the following solvability condition is derived:

$$
\begin{aligned}
& H_{k, 1} K_{22} K_{33} K_{44}-H_{k, 1} K_{22} K_{33} \omega_{k}^{2}-H_{k, 1} K_{22} \omega_{k}^{2} K_{44}+H_{k, 1} K_{22} \omega_{k}^{4} \\
& -9216 H_{k, 1} K_{22} \gamma^{2} \omega_{k}^{2} / 49-H_{k, 1} \omega_{k}^{2} K_{33} K_{44}+H_{k, 1} \omega_{k}^{4} K_{33}+H_{k, 1} \omega_{k}^{4} K_{44} \\
& -H_{k, 1} \omega_{k}^{6}+\frac{343296}{1225} H_{k, 1} \omega_{k}^{4} \gamma^{2}-\frac{2304}{25} H_{k, 1} \gamma^{2} \omega_{k}^{2} K_{44}-\frac{32}{15} \mathrm{i} H_{k, 4} \gamma \omega_{k}^{3} K_{33}-\frac{16}{3} \mathrm{i} H_{k, 2} \gamma \omega_{k}^{3} K_{44} \\
& -\frac{32}{15} \mathrm{i} H_{k, 4} \gamma \omega_{k}^{3} K_{22}+\frac{32}{15} \mathrm{i} H_{k, 4} \gamma \omega_{k}^{5}-\frac{16}{3} \mathrm{i} H_{k, 2} \gamma \omega_{k}^{3} K_{33}-\frac{256}{5} H_{k, 3} \gamma^{2} \omega_{k}^{2} K_{44}+\frac{768}{35} H_{k, 3} \omega_{k}^{4} \gamma^{2} \\
& +\frac{16}{3} \mathrm{i} H_{k, 2} \gamma \omega_{k}^{5}+\frac{1024}{35} H_{k, 3} K_{22} \gamma^{2} \omega_{k}^{2}-\frac{1572864}{1225} \mathrm{i} H_{k, 2} \gamma^{3} \omega_{k}^{3}+\frac{32}{15} \mathrm{i} H_{k, 4} \gamma \omega_{k} K_{22} K_{33} \\
& +\frac{16}{3} \mathrm{i} H_{k, 2} \gamma \omega_{k} K_{33} K_{44}-\frac{786432}{875} \mathrm{i} H_{k, 4} \gamma^{3} \omega_{k}^{3}=0 .
\end{aligned}
$$

The solutions of $A_{1}\left(T_{2}\right)$ and $A_{2}\left(T_{2}\right)$ are expressed as follows:

$$
\left\{\begin{array}{l}
A_{1}\left(T_{2}\right)=\frac{1}{2} a_{1}\left(T_{2}\right) \mathrm{e}^{\mathrm{i} \theta_{1}\left(T_{2}\right)}, \\
A_{1}\left(T_{2}\right)=\frac{1}{2} a_{2}\left(T_{2}\right) \mathrm{e}^{\mathrm{i} \theta_{2}\left(T_{2}\right)},
\end{array}\right.
$$

where $a_{1}\left(T_{2}\right)$ and $a_{2}\left(T_{2}\right)$ are, respectively, the amplitudes of the primary resonance responses, and $\theta_{1}\left(T_{2}\right)$ and $\theta_{2}\left(T_{2}\right)$ are the phase angles of the corresponding responses, respectively. Then, the following four equations are obtained:

$$
\left\{\begin{array}{l}
\Gamma_{11} a_{1}+\Gamma_{12} a_{1}^{2} a_{2} \sin \beta_{1}+\Gamma_{13} b \cos \beta_{2}=0 \\
\Gamma_{21} a_{1} a_{2}^{2}+\Gamma_{22} a_{1} \sigma_{2}+\Gamma_{23} a_{1}^{2} a_{2} \cos \beta_{1}+\Gamma_{24} a_{1}^{3}+\Gamma_{25} b \sin \beta_{2}=0 \\
\Gamma_{31} a_{2}+\Gamma_{32} a_{1}^{3} \sin \beta_{1}=0 \\
\Gamma_{41} a_{2} \sigma_{2}+\Gamma_{42} a_{2} \sigma_{1}+\Gamma_{43} a_{1}^{3} \cos \beta_{1}+\Gamma_{44} a_{1}^{2} a_{2}+\Gamma_{45} a_{2}^{3}=0
\end{array}\right.
$$

where $\Gamma_{11}, \Gamma_{12}, \Gamma_{13}, \Gamma_{21}, \Gamma_{22}, \Gamma_{23}, \Gamma_{24}, \Gamma_{25}, \Gamma_{31}, \Gamma_{32}, \Gamma_{41}, \Gamma_{42}, \Gamma_{43}, \Gamma_{44}$, and $\Gamma_{45}$ are constant coefficients, and

$$
\beta_{1}=T_{2} \sigma_{1}-3 \theta_{1}+\theta_{2}, \quad \beta_{2}=T_{2} \sigma_{2}-\theta_{1} .
$$

The relationships between the detuning parameters and the modal amplitudes are built as 
follows:

$$
\left\{\begin{array}{l}
a_{2}^{2}\left(\Xi_{11} \sigma_{1}-\Xi_{12} \sigma_{2}+\Xi_{13} a_{1}^{2}+\Xi_{14} a_{2}^{2}\right)^{2}+a_{2}^{2} \Xi_{15}=a_{1}^{6}, \\
\left(-\Xi_{21} a_{1}^{2} a_{2}^{2}-\Xi_{22} a_{1}^{2} \sigma_{2}+\Xi_{23} a_{1}^{4}+\Xi_{24} a_{2}^{2} \sigma_{2}\right. \\
\left.-\Xi_{25} a_{2}^{2} \sigma_{1}-\Xi_{26} a_{2}^{4}\right)^{2}+\left(\Xi_{27} a_{1}^{2}+\Xi_{28} a_{2}^{2}\right)^{2}=a_{1}^{2} b^{2},
\end{array}\right.
$$

where $\Xi_{m k}(m=1,2,3,4,5$, and $k=1,2, \cdots, 8)$ are constant coefficients. The stability of the modal steady-state response amplitudes is determined by the Lyapunov stability theory.

\section{Scheme of Galerkin method}

The approximate analytic results are verified by the four-term Galerkin truncation. Therefore, the transverse displacement of the traveling beam is assumed as follows ${ }^{[24-25]}$ :

$$
u(x, t)=\sum_{m=1}^{4} q_{m}(t) \sin (m \pi x), \quad m=1,2,3,4,
$$

where $q_{m}(t)$ ( $\left.m=1,2,3,4\right)$ are the generalized coordinates. Substituting Eq. (38) into Eq. (11), multiplying the resulting equation by the weighting function $\sin (g \pi x)(g=1,2,3,4)$, and integrating the product from 0 to 1 yield

$$
\begin{aligned}
& \ddot{q}_{1}+C \dot{q}_{1}-C_{1} \dot{q}_{2}-C_{2} \dot{q}_{4}+k_{11} q_{1}-k_{12} q_{2}-k_{14} q_{4} \\
& -\alpha A_{3} q_{1}\left(q_{1} \dot{q}_{1}+4 q_{2} \dot{q}_{2}+9 q_{3} \dot{q}_{3}+16 q_{4} \dot{q}_{4}\right)+3 E_{c} \pi^{4} q_{1}\left(q_{1}^{2}+4 q_{2}^{2}+9 q_{3}^{2}+16 q_{4}^{2}\right) / 4 \\
& -\alpha E_{h} \pi^{4} \dot{q}_{1}\left(q_{1}^{2}+4 q_{2}^{2}+9 q_{3}^{2}+16 q_{4}^{2}\right) / 2-12 \alpha E_{h} \pi^{4} q_{2} q_{3} \dot{q}_{4} \\
& +\pi^{4} \alpha E_{h} \gamma q_{2}\left(7 q_{1}^{2} / 3+88 q_{2}^{2} / 21+88 q_{3}^{2} / 7+32608 q_{4}^{2} / 693\right) 16 / 5 \\
& +32 \pi^{4} \alpha E_{h} \gamma q_{4}\left(59 q_{1}^{2} / 21+496 q_{2}^{2} / 63+277 q_{3}^{2} / 11+6016 q_{4}^{2} / 429\right) / 5 \\
& +288 \pi^{4} \alpha E_{h} \gamma q_{1} q_{2} q_{3} / 7+1216 \pi^{4} \alpha E_{h} \gamma q_{1} q_{3} q_{4} / 15-3 \alpha E_{h} \pi^{4} q_{3} \dot{q}_{1} q_{1} / 2 \\
& -12 \alpha E_{h} \pi^{4} q_{3} q_{4} \dot{q}_{2}+3 \alpha E_{h} \pi^{4} q_{1}^{2} \dot{q}_{1} / 4-\alpha E_{h} \pi^{4} 4 q_{2} \dot{q}_{4} q_{1} \\
& -3 \alpha E_{h} \pi^{4} \dot{q}_{3}\left(q_{1}^{2}+4 q_{2}^{2}+16 q_{2} q_{4}\right) / 4-6 \alpha E_{h} \pi^{4} q_{2} q_{3} \dot{q}_{2}-\alpha E_{h} \pi^{4} 4 q_{4} \dot{q}_{2} q_{1} \\
& -4 \alpha E_{h} \pi^{4} q_{2} q_{4} \dot{q}_{1}+24 E_{c} \pi^{4}\left(12 q_{2}^{2} q_{3}+3 q_{1}^{2} q_{3}+48 q_{2} q_{3} q_{4}+16 q_{1} q_{2} q_{4}-q_{1}^{3}\right)-b \sin (\omega t)=0, \\
& \ddot{q}_{2}+16 C \dot{q}_{2}+C_{1} \dot{q}_{1}-C_{3} \dot{q}_{3}+k_{22} q_{2}-k_{23} q_{3}+k_{21} q_{1} \\
& -4 \alpha E_{h} \pi^{4} q_{2}\left(q_{1} \dot{q}_{1}+4 q_{2} \dot{q}_{2}+9 q_{3} \dot{q}_{3}+16 q_{4} \dot{q}_{4}\right)+3 E_{c} \pi^{4} q_{2}\left(q_{1}^{2}+4 q_{2}^{2}+9 q_{3}^{2}+16 q_{4}^{2}\right) \\
& -2 \alpha E_{h} \pi^{4} \dot{q}_{2}\left(q_{1}^{2}+4 q_{2}^{2}+9 q_{3}^{2}+16 q_{4}^{2}\right)+3 E_{c} \pi^{4} q_{1}^{2} q_{4} \\
& +8 \pi^{4} \alpha \gamma E_{h} q_{3}\left(117 q_{1}^{2} / 7+196 q_{2}^{2} / 3+5589 q_{3}^{2} / 77+95408 q_{4}^{2} / 273\right) / 5-18 \alpha E_{h} \pi^{4} q_{3}^{2} \dot{q}_{4} \\
& -36 \alpha E_{h} \pi^{4} q_{3} q_{4} \dot{q}_{3}+8 \pi^{4} \alpha \gamma E_{h} q_{1}\left(q_{1}^{2} / 3+244 q_{2}^{2} / 21+127 q_{3}^{2} / 7+20432 q_{4}^{2} / 693\right) / 5 \\
& -2 \alpha E_{h} \pi^{4} q_{1}^{2} \dot{q}_{4}-12 \alpha E_{h} \pi^{4} q_{1} q_{3} \dot{q}_{4}+\pi^{4} \alpha E_{h} \gamma q_{2} q_{4}\left(19584 q_{3} / 55+7552 q_{1} / 63\right) \\
& -12 \alpha E_{h} \pi^{4} q_{3} q_{4} \dot{q}_{1}+3 E_{c} \pi^{4}\left(6 q_{1} q_{3} q_{4}+3 q_{1} q_{2} q_{3}+9 q_{3}^{2} q_{4}-2 q_{2}^{3}\right)-12 \alpha E_{h} \pi^{4} q_{1} q_{4} \dot{q}_{3} \\
& -4 \alpha E_{h} q_{1} \pi^{4} q_{4} \dot{q}_{1}-6 \alpha \pi^{4} E_{h} q_{1} q_{3} \dot{q}_{2}+4 \alpha E_{h} \pi^{4} q_{2}^{2} \dot{q}_{2}-6 \alpha E_{h} \pi^{4} q_{1} q_{2} \dot{q}_{3} \\
& -6 \alpha E_{h} \pi^{4} q_{2} q_{3} \dot{q}_{1}=0 \text {, }
\end{aligned}
$$




$$
\begin{aligned}
& \ddot{q}_{3}+81 C \dot{q}_{3}+C_{3} \dot{q}_{2}-C_{4} \dot{q}_{4}+k_{33} q_{3}-k_{34} q_{4}-9 \alpha E_{h} \pi^{4} q_{3}\left(q_{1} \dot{q}_{1}+4 q_{2} \dot{q}_{2}+9 q_{3} \dot{q}_{3}+16 q_{4} \dot{q}_{4}\right) \\
& +27 E_{c} \pi^{4} q_{3}\left(q_{1}^{2}+4 q_{2}^{2}+9 q_{3}^{2}+16 q_{4}^{2}\right) / 4-9 \alpha E_{h} \pi^{4} \dot{q}_{3}\left(q_{1}^{2}+4 q_{2}^{2}+9 q_{3}^{2}+16 q_{4}^{2}\right) / 2 \\
& -6 \alpha E_{h} q_{1} \pi^{4} q_{2} \dot{q}_{2}+16 \pi^{4} \alpha E_{h} \gamma q_{2}\left(9 q_{1}^{2} / 7-8 q_{2}^{2} / 3+2403 q_{3}^{2} / 77+2272 q_{4}^{2} / 91\right) / 5+k_{32} q_{2} \\
& +32 \pi^{4} \alpha E_{h} \gamma q_{4}\left(67 q_{1}^{2} / 7+3312 q_{2}^{2} / 77+7479 q_{3}^{2} / 91+1664 q_{4}^{2} / 21\right) / 5+243 / 4 \alpha E_{h} \pi^{4} q_{3}^{2} \dot{q}_{3} \\
& -12 \alpha E_{h} \pi^{4} q_{1} q_{4} \dot{q}_{2}-36 \alpha E_{h} \pi^{4} q_{2} q_{4} \dot{q}_{3}-3 \alpha E_{h} \pi^{4} q_{1}^{2} \dot{q}_{1} / 4-3 \alpha E_{h} \pi^{4} q_{2}^{2} \dot{q}_{1}-12 \alpha E_{h} \pi^{4} q_{1} q_{2} \dot{q}_{4} \\
& -36 \alpha E_{h} q_{3} \pi^{4} q_{4} \dot{q}_{2}+\pi^{4} \alpha E_{h} \gamma q_{1} q_{3}\left(3552 q_{2} / 35+1344 q_{4} / 11\right)-b \sin (\omega t) / 3+9 E_{c} \pi^{4} q_{2}^{2} q_{1} / 2 \\
& -36 \alpha E_{h} q_{2} \pi^{4} q_{3} \dot{q}_{4}-12 \alpha E_{h} \pi^{4} q_{2} q_{4} \dot{q}_{1} \\
& +3 E_{c} \pi^{4} q_{1}^{3} / 8+3 E_{c} \pi^{4}\left(18 q_{2} q_{3} q_{4}-81 q_{3}^{3} / 8+6 q_{1} q_{2} q_{4}\right)=0, \\
& \ddot{q}_{4}+256 C \dot{q}_{4}+C_{2} \dot{q}_{1}+C_{4} \dot{q}_{3}+k_{44} q_{4}+k_{41} q_{1}+k_{43} q_{3}+12 E_{c} \pi^{4} q_{4}\left(q_{1}^{2}+4 q_{2}^{2}+9 q_{3}^{2}+16 q_{4}^{2}\right) \\
& -16 \alpha E_{h} \pi^{4} q_{4}\left(q_{1} \dot{q}_{1}+4 q_{2} \dot{q}_{2}+9 q_{3} \dot{q}_{3}+16 q_{4} \dot{q}_{4}\right)-8 \alpha E_{h} \pi^{4} \dot{q}_{4}\left(q_{1}^{2}+4 q_{2}^{2}+9 q_{3}^{2}+16 q_{4}^{2}\right) \\
& +16 \pi^{4} \alpha E_{h} \gamma q_{3}\left(11 q_{1}^{2} / 21-1332 q_{2}^{2} / 77-2673 q_{3}^{2} / 91+1713 q_{4}^{2} / 21\right) / 5+3 E_{c} \pi^{4} q_{1}^{2} q_{2} \\
& +16 \pi^{4} \alpha E_{h} \gamma q_{1}\left(-13 q_{1}^{2} / 21+428 q_{2}^{2} / 63+511 q_{3}^{2} 11+22288 q_{4}^{2} / 429\right) / 5+18 q_{1} q_{2} q_{3} E_{c} \pi^{4} \\
& +192 \alpha E_{h} \pi^{4} q_{4}^{2} \dot{q}_{4}+\pi^{4} \alpha E_{h} \gamma q_{2} q_{4}\left(2521 q_{1} / 1155+547 q_{3} / 91\right) 256 / 3-2 \alpha E_{h} \pi^{4} \dot{q}_{2}\left(q_{1}+3 q_{3}\right)^{2} \\
& -12 \alpha E_{h} \pi^{4} q_{2} \dot{q}_{3}\left(q_{1}+3 q_{3}\right)-4 \alpha E_{h} \pi^{4} \dot{q}_{1} q_{2}\left(q_{1}+3 q_{3}\right)+3\left(9 q_{3}^{2} q_{2}-32 q_{4}^{3}\right) E_{c} \pi^{4}=0,
\end{aligned}
$$

where

$$
\left\{\begin{array}{l}
C=\alpha\left(E_{b}-E_{a}\right) \pi^{4}, \quad C_{1}=16 \gamma / 5, \quad C_{4}=96 \gamma / \pi \\
A_{1}=\pi^{4} \alpha \gamma E_{b}, \quad A_{2}=\pi^{4} E_{h} \gamma, \quad A_{3}=\pi^{4} E_{h}, \quad E_{h}=\left(E_{c}-E_{d}\right), \\
k_{12}=128 A_{1} / 5, \quad k_{34}=12288 A_{1} / 7, \quad k_{41}=16 A_{1} / 15, \quad k_{21}=8 A_{1} / 3 \\
k_{32}=384 A_{1} / 5, \quad k_{43}=3888 A_{1} / 7 .
\end{array}\right.
$$

The set of the second-order ordinary differential equation (39) is numerically calculated by the fourth-order Runge-Kutta method. In the following numerical examples, the time step is set as $0.001^{[26-27]}$. Moreover, the initial conditions are set as follows:

$$
q_{1}(t)=0.001, \quad q_{m}(t)=0, \quad m=2,3,4, \quad \dot{q}_{m}(t)=0, \quad m=1,2,3,4 .
$$

\section{Numerical results}

Figure 3 illustrates the comparisons of the steady-state periodic responses by use of the multi-scale method and based on the Galerkin method. Similar comparisons with the weaker flexural stiffness of the traveling beam, i.e., $E_{b}=0.03$, are shown in Fig. 4 . Both Figs. 3 and 4 clearly depict that the second-order mode resonates when the excitation frequency is close to the first-order natural frequency. Therefore, the energy transfers from the second-order mode to the first-order mode. Figures 3 and 4 also demonstrate that the approximate analytical results and the numerical results are almost overlapping. Specially, the amplitude curves of resonance at the first-order mode based on the two approaches overlap completely. Compared with the results without the internal resonance in Refs. [10] and [21], the amplitude-frequency curves are 


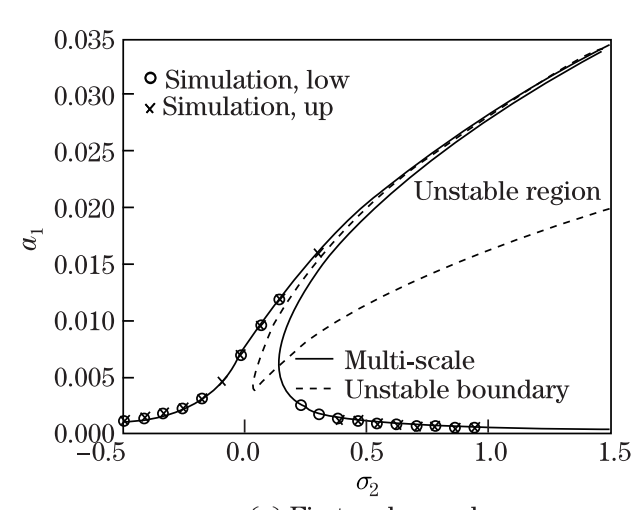

(a) First-order mode

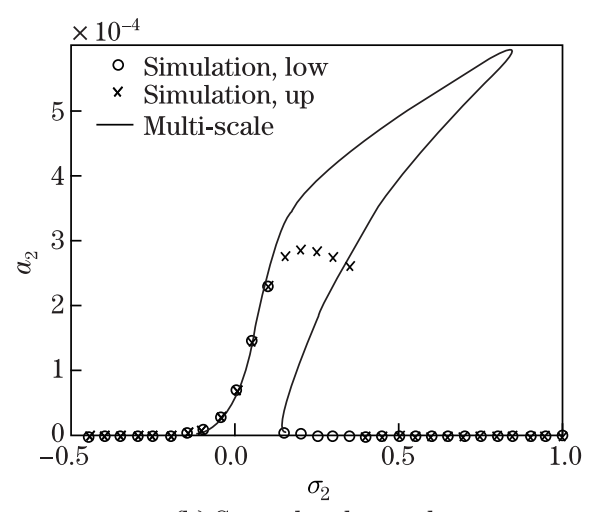

(b) Second-order mode

Fig. 3 Comparison of analytical and Galerkin method's results

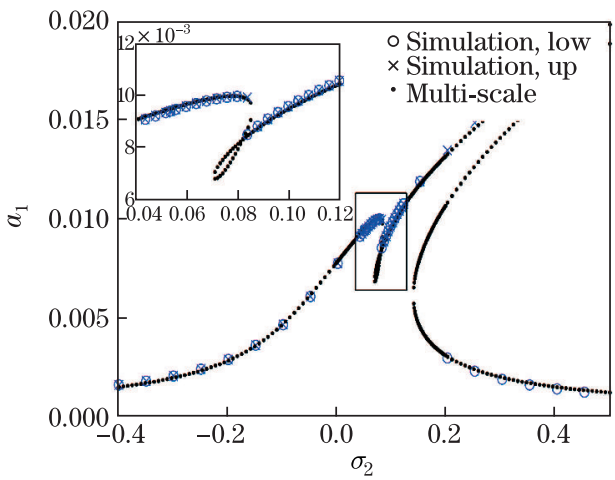

(a) First-order mode

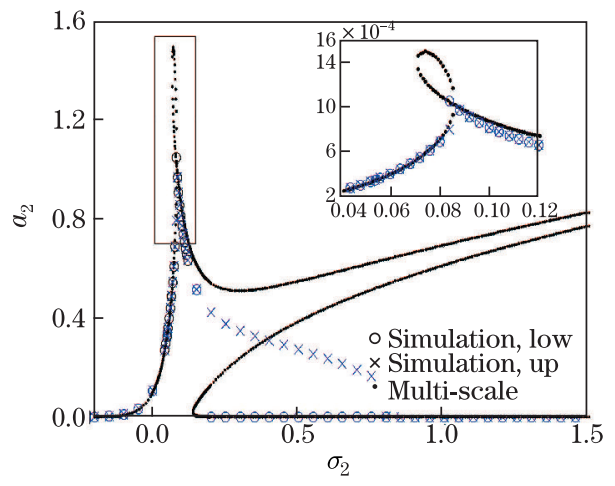

(b) Second-order mode

Fig. 4 Comparison of analytical and Galerkin method's results with weaker flexural stiffness

more complicate in the presence of the internal resonance, specially when the flexural stiffness of the beam is relatively soft.

Figure 5 shows the effects of the amplitude of the excitation on the primary resonance response with the internal resonance. The numerical results show the hysteresis phenomenon of

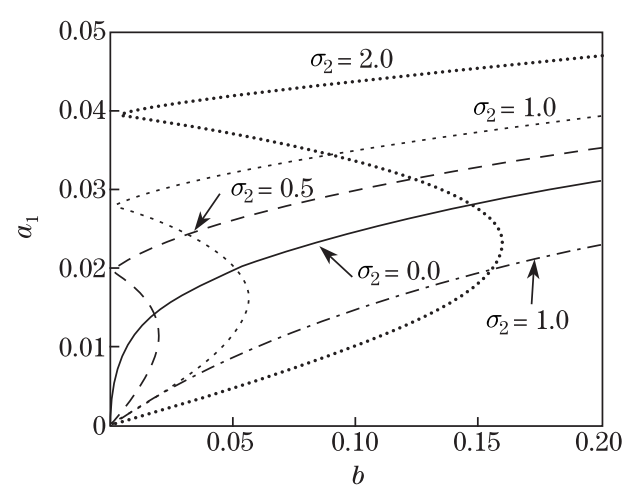

(a) First-order mode

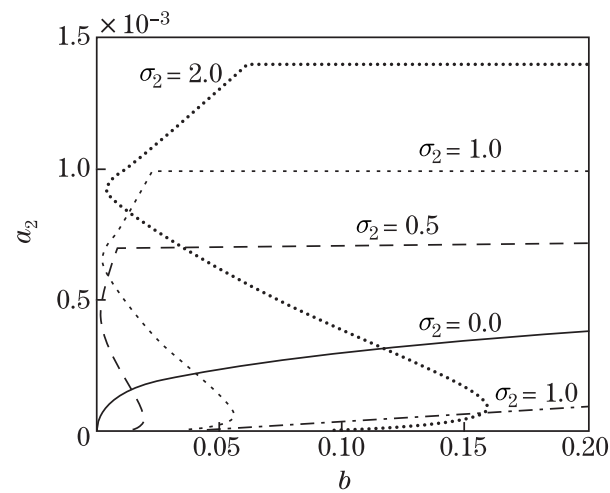

(b) Second-order mode

Fig. 5 Effects of excitation amplitude on resonance responses 
the axially traveling beam. Under the conditions of 3:1 internal resonance between the first two modes and the first- and second-order mode resonates, the amplitude of the resonance response changes with the excitation amplitude. Moreover, the saturated phenomenon is observed in Fig. 5(b). This is an interesting phenomenon since the saturation of the second-order mode happens at the first-order primary resonance.

The effects of the system parameters on the amplitude-frequency curves are presented in Figs. 6, 7, and 8. The results indicate that the amplitudes of the resonance of the first two modes are very sensitive to the system parameters. From Fig. 6, one can easily find that the amplitude-frequency curves bend to the right with the increase in the nonlinear coefficient. The simulations shown in Figs. 7 and 8 depict that the amplitude of the resonance with the internal resonance increases with the decreases in the flexural stiffness and the viscous coefficient of the beam. Moreover, the nonlinear dynamics of the axially traveling beam becomes more complicate when the flexural stiffness and viscous coefficient of the beam become smaller.

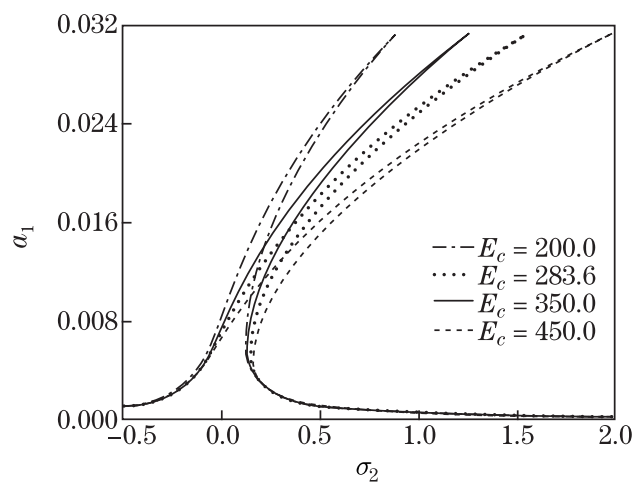

(a) First-order mode

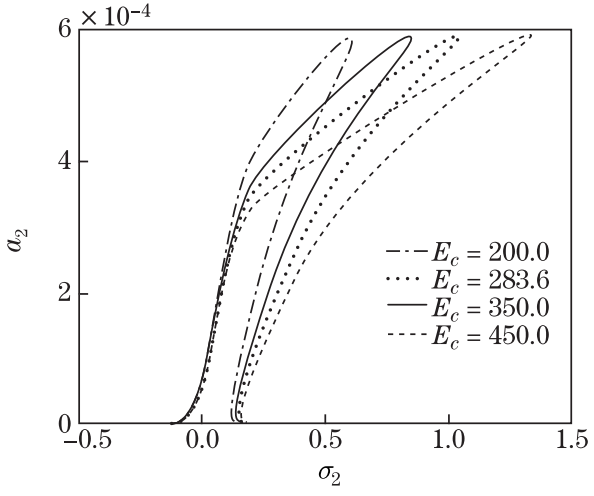

(b) Second-order mode

Fig. 6 Effects of nonlinearity on resonance responses

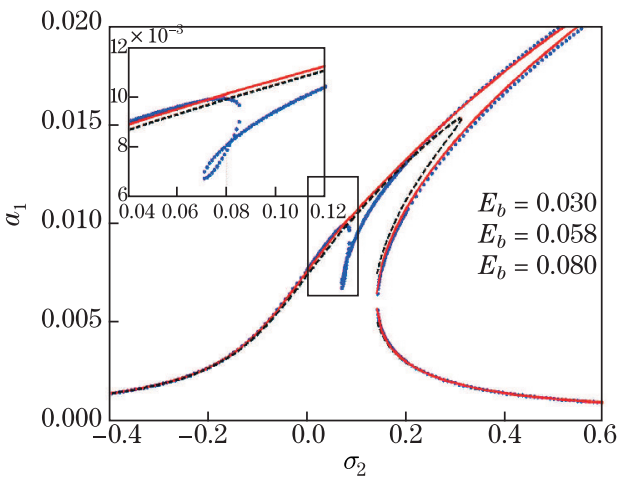

(a) First-order mode

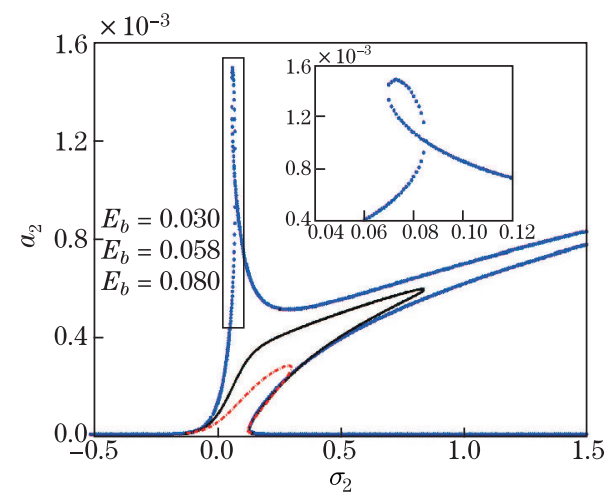

(b) Second-order mode

Fig. 7 Effects of flexural stiffness of traveling beam on resonance responses

\section{Conclusions}

The purpose of this paper is to investigate the effects of the internal resonance and the viscoelastic behaviors on the primary response of the traveling viscoelastic beam. The standard 


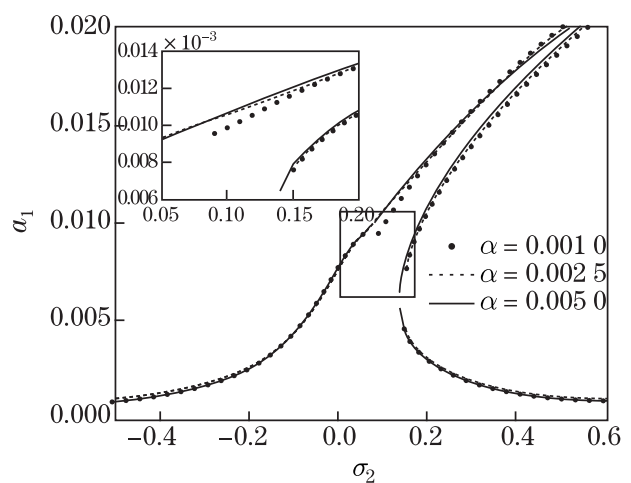

(a) First-order mode

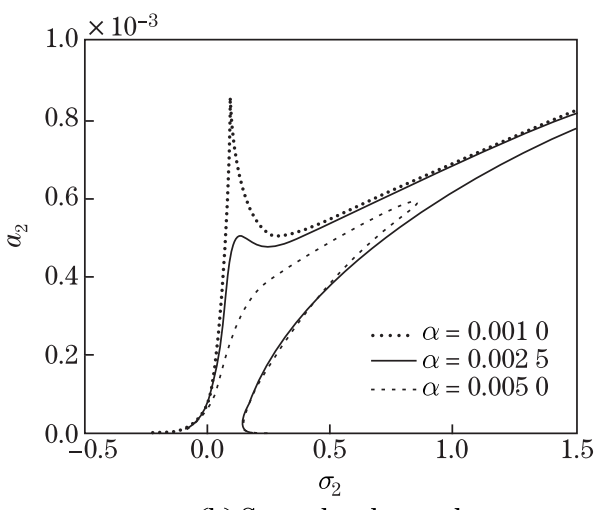

(b) Second-order mode

Fig. 8 Effects of viscous coefficient on resonance responses

linear solid model with the material time derivative is adopted to describe the viscoelastic properties of the axially traveling beam. The real modal functions of the linear derived equation are approximately established. Usually, the linear derived system of the nonlinear governing equation is solved by complex modal functions. The steady-state response amplitudes are firstly determined by the direct multi-scale method with the real modal functions. Then, the Galerkin method is used to verify the approximate solutions. The verification of the Galerkin method shows that the approximate analytical results are acceptable. Therefore, the direct multi-scale method with approximate real modal functions is a reliable approach for studying the nonlinear vibration of the traveling beam. The steady-state responses for the internal resonance modes show that the effect of the internal resonance cannot be ignored.

Open Access This article is distributed under the terms of the Creative Commons Attribution 4.0 International License (http://creativecommons.org/licenses/by/4.0/), which permits use, duplication, adaptation, distribution and reproduction in any medium or format, as long as you give appropriate credit to the original author(s) and the source, provide a link to the Creative Commons license and indicate if changes were made.

\section{References}

[1] Zhu, W. D., Guo, B. Z., and Mote, C. D. Stabilization of a translating tensioned beam through a pointwise control force. Journal of Dynamic Systems Measurement and Control-Transactions of the ASME, 122, 322-331 (2000)

[2] Yang, T. Z. and Yang, X. D. Exact solution of supercritical axially moving beams: symmetric and anti-symmetric configurations. Archive of Applied Mechanics, 83, 899-906 (2013)

[3] Sandilo, S. H. and van Horssen, W. T. On boundary damping for an axially moving tensioned beam. Journal of Vibration and Acoustics-Transactions of the ASME, 134, 011005 (2012)

[4] Marynowski, K. and Kapitaniak, T. Dynamics of axially moving continua. International Journal of Mechanical Sciences, 81, 26-41 (2014)

[5] Yao, M. H. and Zhang, W. Multi-pulse homoclinic orbits and chaotic dynamics in motion of parametrically excited viscoelastic moving belt. International Journal of Nonlinear Sciences and Numerical Simulation, 6, 37-45 (2005)

[6] Ozhan, B. B. and Pakdemirli, M. A general solution procedure for the forced vibrations of a continuous system with cubic nonlinearities: primary resonance case. Journal of Sound and Vibration, 325, 894-906 (2009)

[7] Ozhan, B. B. and Pakdemirli, M. Effect of viscoelasticity on the natural frequencies of axially moving continua. Advances in Mechanical Engineering, 5, 169598 (2013) 
[8] Yang, X. D. and Zhang, W. Nonlinear dynamics of axially moving beam with coupled longitudinaltransversal vibrations. Nonlinear Dynamics, 78, 2547-2556 (2014)

[9] Ding, H. and Zu, J. W. Steady-state responses of pulley-belt systems with a one-way clutch and belt bending stiffness. Journal of Vibration and Acoustics-Transactions of the ASME, 136, 041006 (2014)

[10] Yan, Q. Y., Ding, H., and Chen, L. Q. Nonlinear dynamics of axially moving viscoelastic Timoshenko beam under parametric and external excitations. Applied Mathematics and Mechanics (English Edition), 36(8), 971-984 (2015) DOI 10.1007/s10483-015-1966-7

[11] Tang, Y. Q., Zhang, D. B., and Gao, J. M. Parametric and internal resonance of axially accelerating viscoelastic beams with the recognition of longitudinally varying tensions. Nonlinear Dynamics, 83, 401-418 (2016)

[12] Wang, L. H., Hu, Z. D., Zhong, Z., and Ju, J. W. Dynamic analysis of an axially translating viscoelastic beam with an arbitrarily varying length. Acta Mechanica, 214, 225-244 (2010)

[13] Chen, L. Q. and Chen, H. Asymptotic analysis on nonlinear vibration of axially accelerating viscoelastic strings with the standard linear solid model. Journal of Engineering Mathematics, 67, 205-218 (2010)

[14] Wang, B. Asymptotic analysis on weakly forced vibration of axially moving viscoelastic beam constituted by standard linear solid model. Applied Mathematics and Mechanics (English Edition), 33(6), 817-828 (2012) DOI 10.1007/s10483-012-1588-8

[15] Saksa, T. and Jeronen, J. Dynamic analysis for axially moving viscoelastic Poynting-Thomson beams. Mathematical Modeling and Optimization of Complex Structures, 40, 131-151 (2016)

[16] Ghayesh, M. H. and Amabili, M. Nonlinear stability and bifurcations of an axially moving beam in thermal environment. Journal of Vibration and Control, 21, 2981-2994 (2015)

[17] Sze, K. Y., Chen, S. H., and Huang, J. L. The incremental harmonic balance method for nonlinear vibration of axially moving beams. Journal of Sound and Vibration, 281, 611-626 (2005)

[18] Huang, J. L., Su, R. K. L., Li, W. H., and Chen, S. H. Stability and bifurcation of an axially moving beam tuned to three-to-one internal resonances. Journal of Sound and Vibration, 330, 471-485 (2011)

[19] Wang, L. H., Ma, J. J., Yang, M. H., Li, L. F., and Zhao, Y. Y. Multimode dynamics of inextensional beams on the elastic foundation with two-to-one internal resonances. Journal of Applied Mechanics-Transactions of the ASME, 80, 061016 (2013)

[20] Sahoo, B., Panda, L. N., and Pohit, G. Combination, principal parametric and internal resonances of an accelerating beam under two frequency parametric excitation. International Journal of NonLinear Mechanics, 78, 35-44 (2016)

[21] Ding, H., Zhang, G. C., Chen, L. Q., and Yang, S. P. Forced vibrations of supercritically transporting viscoelastic beams. Journal of Vibration and Acoustics-Transactions of the ASME, 134, 051007 (2012)

[22] Liu, D., Xu, W., and $\mathrm{Xu}, \mathrm{Y}$. Dynamic responses of axially moving viscoelastic beam under a randomly disordered periodic excitation. Journal of Sound and Vibration, 331, 4045-4056 (2012)

[23] Ding, H. and Zu, J. W. Periodic and chaotic responses of an axially accelerating viscoelastic beam under two-frequency excitations. International Journal of Applied Mechanics, 5, 1350019 (2013)

[24] Kang, H. J., Zhao, Y. Y., and Zhu, H. P. Out-of-plane free vibration analysis of a cable-arch structure. Journal of Sound and Vibration, 332, 907-921 (2013)

[25] Ding, H. Steady-state responses of a belt-drive dynamical system under dual excitations $A C T A$ Mechanics Sinica, 32, 156-169 (2016)

[26] Zhang, J. R., Guo, Z. X., Zhang, Y., Tang, L., and Guan, X. Inner structural vibration isolation method for a single control moment gyroscope. Journal of Sound and Vibration, 361, 78-98 (2016)

[27] Li, X. H. and Hou, J. Y. Bursting phenomenon in a piecewise mechanical system with parameter perturbation in stiffness. International Journal of Non-Linear Mechanics, 81, 165-176 (2016) 